\section{Nociception, douleur et autisme}

\author{
Lucien Ruelle-Le Glaunec ${ }^{1,2}$, Perrine Inquimbert ${ }^{1,2}$, \\ Sylvain Hugel ${ }^{1}$, Rémy Schlichter ${ }^{1,2}$, Jean-Louis Bossu ${ }^{1}$
}

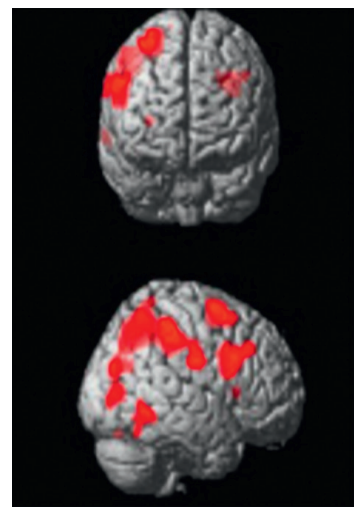

${ }^{1}$ CNRS, 5 rue Blaise-Pascal 67000 Strasbourg, France. ${ }^{2}$ Université de Strasbourg, Institut des neurosciences cellulaires et intégratives, UPR 3212, 8 allée du Général Rouvillois, F-67000 Strasbourg, France.

jlbossu@inci-cnrs.unistra.fr

les modalités sensorielles (visuelles, auditives, olfactives, gustatives, somato-sensorielles) [2]. Il peut s'agir d'hyper-ou d'hypo-réactivité, ou d'un intérêt atypique pour des informations sensorielles provenant de l'environnement [1]. L'intégration multisensorielle, essentielle pour la perception et la compréhension d'informations sociales complexes, est également vraisemblablement affectée chez les personnes souffrant de TSA [2]. Cette revue s'intéresse à une fonction spécifique de la modalité somato-sensorielle, la nociception. Ce terme regroupe les mécanismes de détection et d'encodage des stimulus potentiellement nocifs pour l'organisme, qui, après interprétation consciente et subjective, peuvent donner lieu à la sensation de douleur. Étudier ces aspects dans le cadre de l'autisme semble fondamental. Les patients porteurs de TSA sont en effet plus susceptibles d'être confrontés à des situations douloureuses au cours de leur vie que la population générale [3]. Ils présentent ainsi fréquemment des comorbidités pouvant être douloureuses, telles que l'épilepsie ou des troubles gastro-intestinaux, qui les conduisent à subir plus fréquemment que dans la population générale, des interventions médicales elles aussi potentiellement douloureuses [3]. Certains sujets autistes manifestent de plus des comportements à caractère automutilateur [4]. Appréhender la douleur, qu'elle soit aiguë ou chronique, chez ces personnes, aussi bien dans son aspect sensoriel qu'affectif, et son impact sur le plan psychologique, apparaît donc être un volet essentiel dans la prise en charge de cette pathologie.

\section{La douleur et les voies nociceptives}

La douleur est définie par l'International Association for the Study of Pain comme une expérience sensorielle et émotionnelle désagréable en réponse à une lésion tissulaire réelle ou potentielle, ou décrite en de tels termes. Elle résulte de l'interprétation consciente et subjective de I'information nociceptive. 


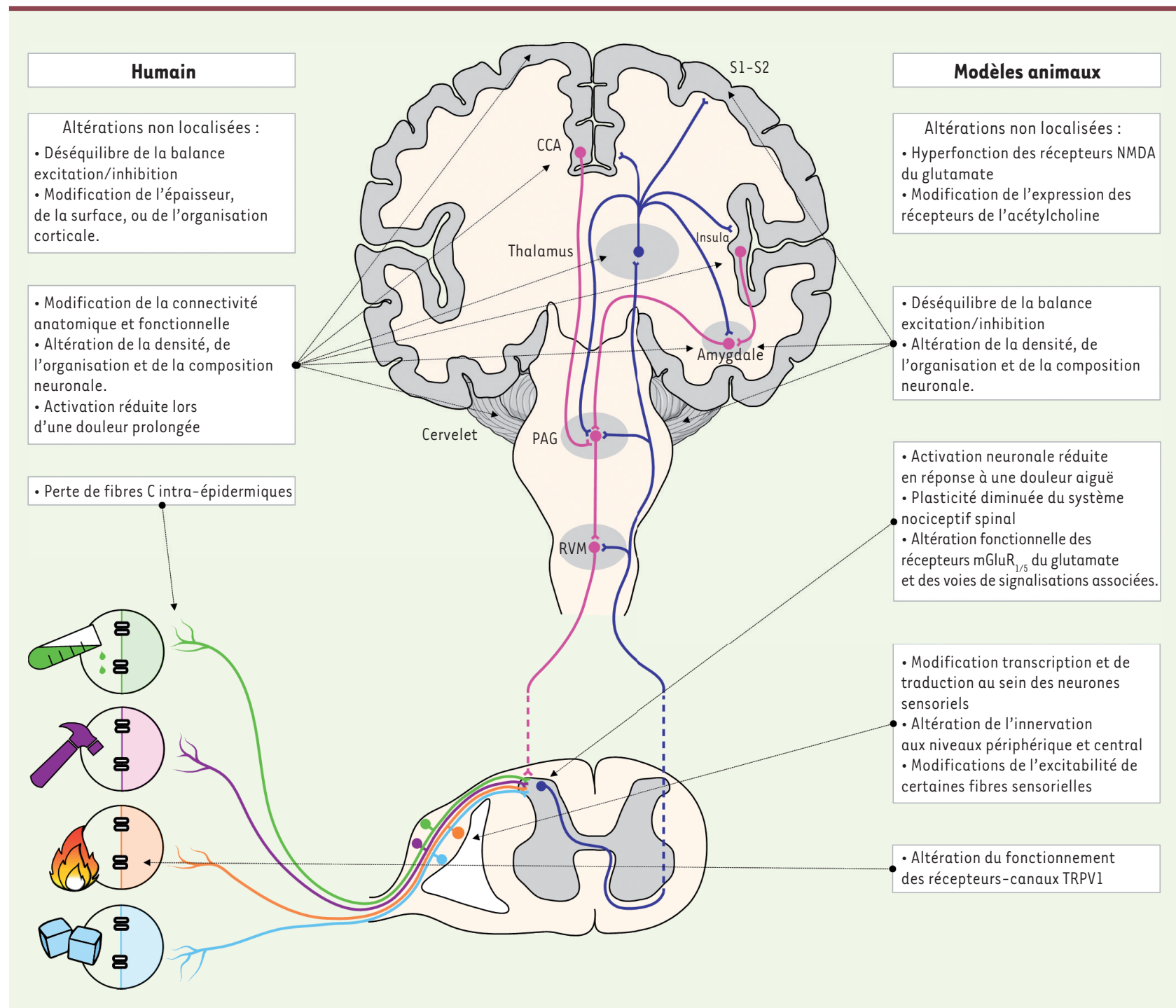

Figure 1. Les particularités de la sensibilité nociceptive dans l'autisme: hypothèses cellulaires et moléculaires. Les nocicepteurs détectent les stimulus nocifs en périphérie et transmettent l'information aux neurones du réseau de la corne dorsale de la moelle épinière. L'information qui en résulte est transmise à l'encéphale et notamment au thalamus via la voie spinothalamique. S'ensuit un traitement cérébral complexe mettant en jeu différentes structures (S1-S2 : cortex somato-sensoriel, CCA : cortex cingulaire antérieur, insula, amygdale, cervelet, etc.), donnant potentiellement lieu à la sensation de douleur. En retour, les structures cérébrales envoient des projections sur la substance grise péri-aqueducale (PAG) et sur la réticulée ventro-médiane (RVM), deux structures modulant, via les terminaisons de leur neurones, l'intégration spinale du message nociceptif. Certaines altérations présentes chez les sujets TSA semblent être non localisées et communes à d'autres désordres non strictement nociceptifs. Cependant, des changements morphologiques et fonctionnels à différents niveaux des voies nociceptives sont décrits. Ils peuvent concerner la transduction des stimulus potentiellement nocifs au niveau périphérique, l'intégration nociceptive spinale et l'élaboration de la sensation de douleur au niveau cérébral.

Schématiquement (Figure 1), le message nociceptif est initialement produit par l'activation de neurones sensoriels dits «nocicepteurs » innervant la périphérie (peau, muscles, etc.), ainsi que les organes internes [5]. Ces nocicepteurs, selon leurs modalités d'activation, détectent les stimulus nocifs, qu'ils soient thermiques, mécaniques ou chimiques. Les protéines responsables de la transduction de ces stimulus en signal électrique sont plus ou moins spécifiques d'une modalité et conduisent à la dépolarisation de la terminaison périphérique et à la genèse de potentiels d'action. Un nombre important de ces protéines a été décrit, comme par exemple les canaux TRPVI (transient receptor potential vanilloid 1) pour la nociception au chaud, ou TRPM8 (transient receptor potential cation channel subfamily Melastatin member 8) pour la perception du froid [6]. L'information nociceptive résultante est codée sous la forme de trains de potentiels d'action, dont la fréquence dépend 
de l'intensité de la stimulation [6]. Elle est ensuite acheminée via les racines dorsales à la corne dorsale de la moelle épinière. L'intégration des messages nociceptifs à ce niveau, avant leur transmission vers les centres supérieurs, est un processus complexe qui met en jeu un dense réseau d'interneurones constitués d'environ $75 \%$ de neurones excitateurs et $25 \%$ de neurones inhibiteurs [7]. L'équilibre entre la composante excitatrice et inhibitrice constitue un élément capital dans l'intégration et le filtrage des entrées nociceptives. L'information est ensuite transmise à des neurones de projection dont les axones projettent vers des régions supra-spinales. Des changements plastiques au sein de ce circuit ont été décrits comme pouvant entraîner des hypo- ou des hyperalgésies (des douleurs diminuées ou augmentées pour un stimulus normalement douloureux) et des allodynies (des douleurs provoquées par un stimulus normalement indolore) $[5,7]$. Le message nociceptif est modulé au cours de son trajet de la périphérie vers le cortex cérébral. Cette modulation est particulièrement importante dans la corne dorsale de la moelle épinière. Deux types de modulations peuvent être distingués à ce niveau. L'un repose sur les fibres sensorielles tactiles non nociceptives, et est décrit par le concept du «gate control», théorisant l'interaction entre les voies tactile et nociceptive $[5,7]$, et rendant compte de l'observation qu'un léger toucher peut diminuer la sensation de douleur aiguë. L'autre met en jeu des voies descendantes provenant de nombreuses régions supraspinales et, notamment, du tronc cérébral [8]. Ces voies exercent, via différents médiateurs (GABA, glutamate, sérotonine, noradrénaline, dopamine, enképhalines, etc.), une modulation de l'intégration des informations nociceptives au niveau spinal [8].

Après cette intégration spinale complexe, le message nociceptif porté par les neurones de projection atteint l'encéphale par différentes voies. Chez l'homme, il s'agit notamment de la voie spinothalamique [5]. Les informations ascendantes sont intégrées et filtrées au niveau du thalamus, principalement dans les noyaux ventro-postéro-latéraux, -inférieurs et -médians [5]. Les informations sont ensuite transmises à différentes structures cérébrales constituant la «matrice de la douleur » (cortex somato-sensoriel, cortex insulaire, cortex cingulaire antérieur, etc.) $[5,9,10]$. L'interaction fonctionnelle entre ces structures résulte en une sensation/perception globale de douleur dans sa dimension sensori-discriminative, affective et cognitive. Cependant, le rôle exact de chacune de ces structures dans les différents aspects de la sensation/perception douloureuse reste encore à déterminer.

\section{Évaluation de la douleur chez les sujets neurotypiques}

Bien qu'il semble évident qu'évaluer une douleur soit capital lors d'un examen clinique, cela peut parfois s'avérer délicat. Les méthodes utilisées peuvent être directes ou indirectes, objectives ou subjectives, et dépendent de l'âge, de l'état physiologique et psychologique du sujet. Une liste exhaustive des outils disponibles en clinique peut être consultée sur le site de la Société française d'étude et de traitement de la douleur'.

${ }^{1}$ https://www.sfetd-douleur.org/
Le plus souvent, chez l'adulte conscient et capable de s'exprimer, et chez l'enfant âgé de plus de 6 ans, les méthodes utilisées sont directes et subjectives. Elles se fondent principalement sur l'auto-évaluation. Les principales approches sont simples. Elles utilisent des échelles unidimensionnelles, numériques verbales ou visuelles analogiques qui permettent au patient de préciser l'intensité de sa douleur, associées à des schémas corporels pour indiquer sa localisation. Pour les jeunes enfants (entre 4 et 6 ans), les méthodes utilisées chez l'adulte ont été adaptées avec, par exemple, des échelles visuelles analogiques représentant des visages plus ou moins expressifs d'un certain degré de souffrance, ou des schémas corporels grossiers (des bonshommes). Ces méthodes simples permettent une première évaluation de la douleur d'un individu ainsi que le suivi de l'efficacité d'un traitement antalgique, mais elles ne donnent pas ou peu d'informations sur les caractéristiques de la douleur. Des approches plus complexes sont donc parfois utilisées, comme des échelles dites multidimensionnelles, à la fois quantitatives (intensité) et qualitatives (type de douleur, modalité, impact psychologique, etc.). Elles tiennent compte de la composante sensori-discriminative mais aussi affective de la douleur. En France, le questionnaire douleur de l'hôpital Saint-Antoine (DDSA) est l'un des plus utilisés.

II est cependant des cas où ces méthodes ne sont pas appropriées, notamment lorsque la communication est impossible ou que les capacités cognitives nécessaires à l'introspection sont insuffisantes (nouveau-né, déficit intellectuel, état végétatif, etc.). De nombreuses méthodes d'évaluation alternatives ont donc été développées. Elles reposent sur l'observation par le personnel soignant de certains signes cliniques : on parle alors d'hétéro-évaluation.

Chez les nouveau-nés et enfants en bas âge (entre 0 et 3 ans), l'observation des mimiques faciales (visage calme, contractions des paupières, froncements des sourcils, accentuations des sillons naso-labiaux), des mouvements des membres (calmes et doux, présence de pédalages, d'écartements des orteils, membres inférieurs raides ou surélevés, agitations des bras, réactions de retrait) et des expressions vocales (gémissements, cris de longue durée, voire hurlements constants) permettent d'obtenir un score indirect du niveau de douleur.

Chez l'adulte incapable de s'exprimer (par exemple s'il est en état végétatif), l'observation des expressions faciales, de l'état de quiétude, du tonus musculaire, d'éventuelles vocalisations, ainsi que la potentielle adaptation au respirateur, peuvent constituer des indices qui renseignent indirectement sur le niveau de douleur. 
D'autres méthodes de mesure se voulant plus objectives ont été développées. Certaines, comme le test sensoriel quantitatif (QST), utilisent des stimulations thermiques et mécaniques standardisées pour évaluer la modalité somato-sensorielle dans son ensemble. Mais elles nécessitent l'absence de déficience intellectuelle majeure chez le sujet $[3,11]$. Finalement, des outils plus indirects, principalement utilisés en anesthésie, analysent des paramètres autonomes, notamment l'augmentation de l'activité du système nerveux sympathique lors d'une expérience nociceptive. Ainsi, la fréquence cardiaque et sa variabilité, la pression artérielle, le degré de dilatation de la pupille, ou la conductance électrodermale, sont de bons indices du niveau de nociception [12].

\section{Mesurer la douleur chez les sujets autistes}

Du fait des déficits de communication qui caractérisent les sujets autistes, ainsi que des potentiels déficits intellectuels associés, il est très difficile, à part pour l'autisme de haut niveau de fonctionnement, d'évaluer leur douleur en utilisant les tests classiques d'auto-évaluation [13]. Actuellement, aucun protocole permettant de mesurer la douleur chez l'ensemble des patients présentant des TSA n'a été validé. Néanmoins, des échelles d'hétéro-évaluation, validées chez des enfants incapables de communiquer verbalement ou ayant des déficits cognitifs importants, peuvent être utilisées chez certains enfants autistes. Récemment, l'échelle NCCPC (non-communicative children pain checklist) a été validée en français, sous le nom de GED-DI (grille d'évaluation douleur-déficience intellectuelle) [14]. À l'origine, cette grille a été conçue pour quantifier la douleur post-opératoire chez des enfants non communicants. Après quelques ajustements prenant en compte les réactions émotionnelles, l'hyper-vigilance ainsi que les expressions idiopathiques et douloureuses spécifiques des patients porteurs de TSA, elle permettrait également de quantifier la douleur au quotidien chez la plupart des enfants autistes [15]. Cette grille, distribuée aux parents, aux puériculteurs, aux soignants, ou au personnel pédagogique, évalue six catégories d'items: les expressions vocales, l'activité sociale, les expressions faciales, le tonus et la réactivité, ainsi que des signes physiologiques. Comme chez les sujets neurotypiques, les outils de mesure de l'activité du système nerveux autonome peuvent également être utilisés.

\section{La sensibilité douloureuse des sujets autistes}

Auparavant, les enfants autistes étaient considérés comme insensibles car certains présentaient des réactions inhabituelles lors d'une situation douloureuse et s'automutilaient $[3,4]$. Ces observations, associées à certaines descriptions cliniques anecdotiques et quelques récits autobiographiques, ont longtemps laissé penser à une absence de sensibilité à la douleur des sujets autistes [3]. À tel point qu'aujourd'hui, les critères diagnostiques des TSA incluent une apparente insensibilité à la douleur [1].

Pourtant, bien qu'un certain nombre d'éléments suggèrent que la réaction à la douleur des sujets autistes est altérée, par rapport à la population neurotypique, les résultats expérimentaux concernant leur sensibilité ne font pas consensus. Par exemple, lors d'une prise de sang, certaines études mettent en évidence une diminution de réactivité comportementale à la douleur par rapport à un groupe témoin. D'autres, en revanche, révèlent une augmentation et certaines ne démontrent aucune différence $[3,16]$. De manière générale, la sensibilité et les réactions comportementales lors d'une expérience douloureuse sont souvent analysées selon des protocoles très divers. Elles résultent de l'étude de rapports réalisés par les parents ou par les éducateurs spécialisés, ou d'observations faites à la suite d'interventions médicales potentiellement douloureuses (prise de sang, soins dentaires) et lors de tests expérimentaux. Les résultats sont donc hétérogènes. Ils dépendent en grande partie de la méthodologie utilisée ainsi que du contexte dans lequel les évaluations ont été réalisées. Il est important de noter qu'une réactivité comportementale apparaissant comme diminuée chez les sujets porteurs de TSA, par rapport aux témoins, n'indique pas nécessairement une sensibilité moindre, mais un mode d'expression de la douleur potentiellement différent, ayant pour origine des troubles de communication, de représentation du corps, et des déficits intellectuels [16]. Par exemple, lors de la mesure de paramètres indirects plus objectifs, comme l'activité du système nerveux autonome, des augmentations plus importantes de la fréquence cardiaque ${ }^{2}$ lors de la réalisation d'une prise de sang sont retrouvées, indiquant une hyper-sensibilité, alors que l'échelle comportementale, réalisée en parallèle, révèle une hypo-sensibilité [16].

Pour réduire cette hétérogénéité dans les protocoles, des études se voulant plus standardisées utilisent des stimulations nociceptives calibrées, comme dans le test sensoriel quantitatif (Tableau I). La plupart de ces études ne retrouvent aucune différence systématique de sensibilité à la douleur entre les sujets porteurs de TSA et des sujets neurotypiques. Cependant, en distinguant les différentes modalités nociceptives, certaines différences peuvent apparaître, mais elles sont loin d'être consensuelles. Concernant la sensibilité nociceptive thermique au chaud et au froid, par exemple, une hyper-sensibilité mais également une absence de différence ont été décrites [11, 17-22]. Quant aux études relatives à la modalité nociceptive mécanique, elles indiquent des normo-, des hypo- ou des hyper-sensibilités [11, 19, 23, 24].

Ces résultats hétérogènes suggèrent qu'il n'est pas encore possible de répondre à une question globale de différence de sensibilité nociceptive, en termes d'hyper-, de normo- ou d'hypo-sensibilité, entre patients

\footnotetext{
${ }^{2}$ L'augmentation de fréquence cardiaque ne traduit pas formellement une hypersensibilité nociceptive, il peut s'agir d'une conséquence d'un stress.
} 


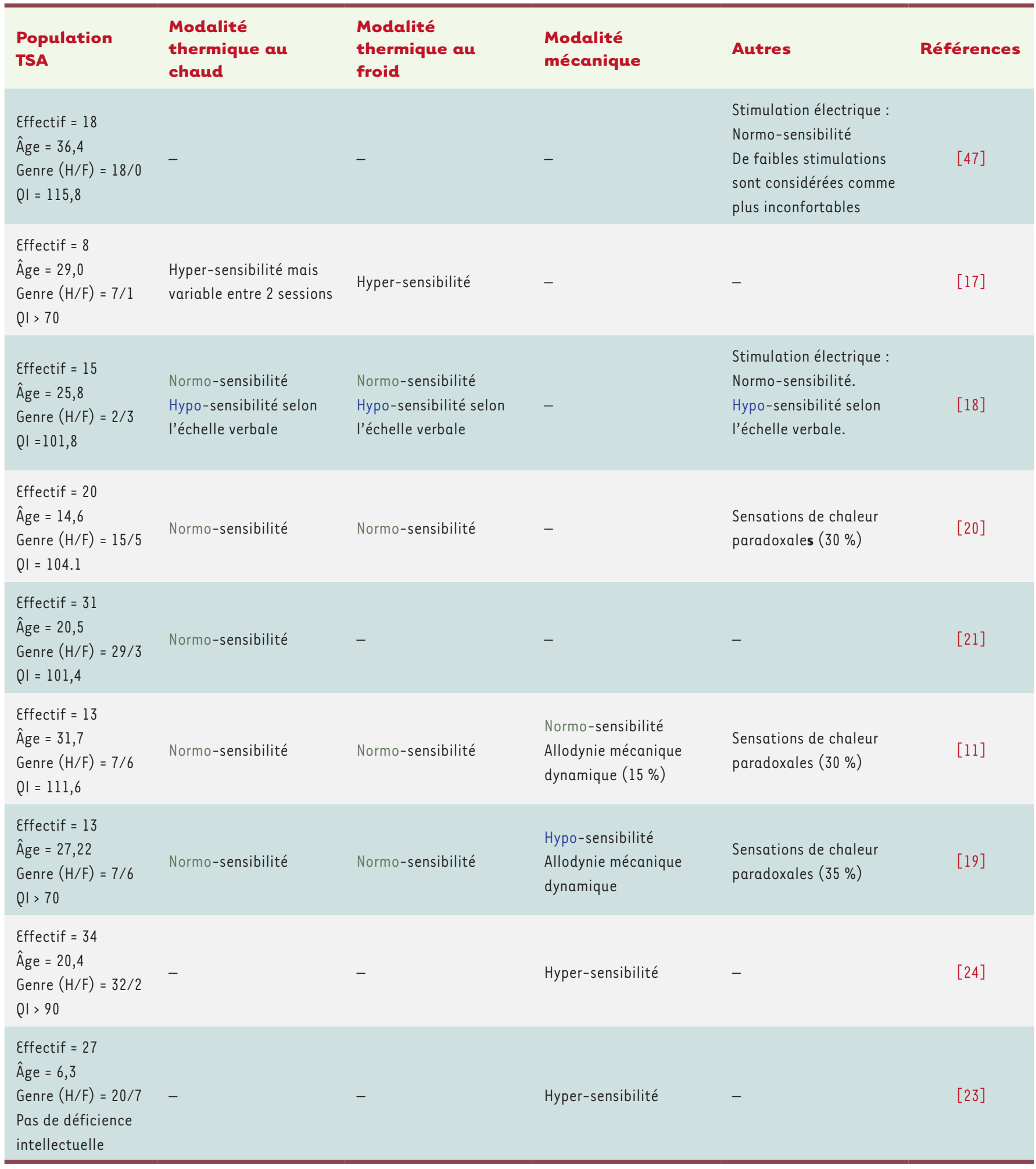

Tableau I. Sensibilité douloureuse des patients TSA. Chez l'homme, la sensibilité douloureuse est souvent mesurée par l'application d'une stimulation d'intensité variable, le sujet indique lorsque celle-ci devient douloureuse. Pour la modalité thermique, le sujet est exposé à une température croissante $\left(32-52^{\circ} \mathrm{C}\right)$ pour le chaud et décroissante $\left(32-0^{\circ} \mathrm{C}\right)$ pour le froid. La sensibilité douloureuse mécanique est mesurée sur le même principe, en appliquant une force croissante à l'aide d'un dynamomètre calibré. La stimulation électrique stimule directement les fibres périphériques de la peau en utilisant un courant sinusoïdal d'intensité croissante. Cette méthode diffère de l'échelle verbale dans laquelle le sujet score sur une échelle numérique l'intensité d'une stimulation donnée. 
porteurs de TSA et population neurotypique. La population de patients est cependant loin d'être homogène. II semble donc que d'autres paramètres nécessitent d'être pris en compte, comme le niveau de déficience intellectuelle, le sexe, la présence de comorbidités ou le contexte environnemental au moment des mesures. Les sujets décrits comme hypo-sensibles seraient en effet ceux qui présentent les symptômes autistiques les plus sévères [4]. Cela est d'autant plus important à prendre en compte que la quasi-totalité des études évaluant la sensibilité douloureuse et nociceptive de ces patients a été réalisée chez des sujets sans déficience intellectuelle. Néanmoins, il est intéressant de noter que certains d'entre eux présentent des sensations paradoxales de chaleur (comme des sensations de chaleur ou de brûlure après stimulations autres que thermiques chaudes) et des allodynies mécaniques dynamiques ${ }^{3}$ que l'on ne retrouve pas chez les sujets témoins $[11,19,20]$. Ces particularités peuvent laisser penser que l'encodage des signaux nociceptifs conduisant à la sensation de douleur pourrait être altéré chez certains sujets autistes.

\section{Les hypothèses chez l'homme}

Les méthodes utilisées habituellement pour évaluer la douleur ne permettent pas toujours de mettre en évidence des différences dans le ressenti des sujets autistes. Cependant, certaines études utilisant des questionnaires multidimensionnels afin d'évaluer différents aspects de la douleur, décrivent une diminution des aspects affectifs et subjectifs, sans modification de l'aspect sensori-discriminatif et du seuil global de sensibilité à la douleur. Ces données suggèrent une potentielle altération des voies ou structures responsable de l'aspect cognitif et affectif de la douleur [18]. Ces méthodes nécessitent de la part du sujet, des capacités introspectives et cognitives intactes, ce qui n'est pas toujours le cas chez les patients porteurs de TSA. L'absence de différences dans la sensibilité douloureuse retrouvée dans certaines études n'indique donc pas obligatoirement une absence de différences d'encodage des stimulations nociceptives. Par exemple, la réponse corticale observée par électroencéphalographie à la suite d'une stimulation nociceptive chaude, appelée potentiel évoqué thermique, est diminuée chez les individus porteurs de TSA, et ceci même si l'intensité n'est pas décrite comme différente sur l'échelle verbale [21]. Récemment, l'imagerie fonctionnelle par IRM (imagerie par résonance magnétique) a permis de caractériser une réponse cérébrale «signature » de la douleur, définie par l'augmentation de l'activité hémodynamique de certaines zones du cerveau (thalamus, cortex somato-sensoriel, insula, cortex cingulaire antérieur, etc.) lors d'une stimulation nociceptive [10]. Initialement, cette réponse cérébrale « signature » de la douleur n'est pas différente chez les sujets porteurs de TSA par rapports aux témoins, mais elle est grandement réduite lors d'une douleur soutenue, alors, qu'une fois encore, l'intensité évaluée à l'aide d'une échelle verbale n'est pas différente [22]. Cette observation suggère que la sensibilité douloureuse n'est pas forcément

${ }^{3}$ Sensation douloureuse déclenchée par un stimulus mécanique dynamique normalement indolore (caresse, frottement des vêtements sur la peau, etc.) altérée, mais que c'est la manière de faire face à une douleur qui pourrait être différente entre les sujets porteurs de TSA et les sujets neurotypiques. Cela pourrait expliquer, en partie, la coexistence des hypo- et hypersensibilités qui sont rapportées [22].

Ainsi, si certains sujets présentant des TSA traitent les informations nociceptives de façon atypique, ces altérations peuvent provenir d'une mauvaise transduction du stimulus potentiellement nocif au niveau périphérique, d'un mauvais codage spinal, d'une altération du traitement cérébral de l'information, voire d'une atypie dans la mise en place des contrôles descendants. En se fondant sur les études réalisées sur d'autres modalités sensorielles (la vision, l'audition, etc.), il ne semble pas que la détection des stimulus soit systématiquement altérée. Les observations suggèrent davantage une modification des processus d'intégration [2]. Concernant la modalité somato-sensorielle, ce point est néanmoins plus difficile à évaluer, étant donné l'absence d'organe précis dédié à cette fonction. Des modifications de sensibilité tactile ont cependant déjà été décrites [25]. Concernant la nociception (Figure 1), certains mécanismes centraux d'encodage des stimulus nociceptifs pourraient être à l'origine d'un traitement atypique de l'information expliquant ainsi les sensations de chaleur paradoxale [11]. Par exemple, des altérations des régions somato-sensorielles corticales et sous-corticales pourraient potentiellement être associées aux phénomènes d'automutilation [4]. En effet, si l'on considère les structures centrales impliquées dans l'élaboration de la sensation de douleur, certaines d'entre elles, telles que le cortex somatosensoriel (aspect sensori-discriminatif), l'amygdale (aspect émotionnel) ou le cervelet, présentent des anomalies chez les sujets autistes [26]. Les changements anatomiques et fonctionnels observés chez les patients semblent également corrélés à d'autres altérations somato-sensorielles, telles que la sensibilité tactile [25]. En effet, au niveau spinal, des phénomènes pouvant correspondre au concept de «gate control», dans lequel les fibres non nociceptives tactiles sont impliquées, pourraient être modifiés chez les sujets porteurs de TSA. Cependant, des stimulations répétitives périphériques non nociceptives (tactiles, vibratoires, etc.) réduiraient la sensibilité douloureuse mécanique de ces patients, démontrant que les mécanismes de type « gate control » restent fonctionnels et pourraient représenter une cible thérapeutique intéressante [27]. II semble donc qu'il puisse exister chez certains sujets autistes des particularités quant à la perception des messages nociceptifs et l'élaboration de la sensation de douleur. Cependant, en raison de l'hétérogénéité des 
résultats obtenus chez l'homme, il est difficile de conclure quant aux mécanismes impliqués. Pour s'affranchir de certains biais dans l'évaluation de la douleur (subjectivité, influence par des facteurs cognitifs), pour réaliser des mesures standardisées, et pour caractériser les mécanismes cellulaires et moléculaires impliqués, avoir recours à des modèles animaux pourrait être envisagé.

\section{L'apport des modèles animaux}

La plupart des études réalisées chez l'animal utilisent le rongeur. Parmi les modèles fréquemment exploités, on retrouve des modèles génétiques dans lesquels différents gènes qui ont été associés aux TSA humains (Shank3, Shank2, Chd8 [chromodomain helicase DNA binding protein 8], PTEN [phosphatase and Tensin homolog], Admp [antidorsalizing morphogenetic protein]), ou des gènes syndromiques de troubles neurodéveloppementaux (Mecp2, Fmrl, Tsc] [TSC complex subunit 1], Tsc2, Syngapl [synaptic Ras GTPase activating protein 1], Ube3a [ubiquitin protein ligase $\varepsilon 3 A]$ ), ont été mutés ou invalidés [28-31]. Des modèles environnementaux sont également disponibles. Il sont obtenus par exposition in utero à des agents inflammatoires, comme les lipopolysaccharides bactériens (LPS) ou l'acide polyinosinique:polycytidylique (Poly I:C) [30], ou des agents tératogènes, tels que le valproate de sodium (VPA) [32-34] $(\rightarrow)$.

$(\rightarrow)$ Voir la Synthèse

de J.L. Bossu et

S. Roux, $m / s n^{\circ} 3$, Certaines souches murines consanguines, comme mars 2019, page 236 les souris $\mathrm{BTBR}^{4}$, sont également utilisées $[30,35]$. Ces modèles animaux ont été validés par l'utilisation de tests d'observation révélant des déficits de comportements sociaux ainsi que la présence de comportements stéréotypés, reproduisant en partie les symptômes des TSA. Du point de vue des particularités somato-sensorielles, des anomalies de la sensibilité tactile ont été mises en évidence dans certains de ces modèles animaux, et indiquent des altérations périphériques et centrales [25].

Ces modèles de TSA présentent un intérêt pour l'étude des anomalies nociceptives liées à l'autisme. Les résultats semblent néanmoins dépendre de la modalité nociceptive testée (chaud, froid, mécanique, viscérale) (Tableau II), mais un consensus apparaît en ce qui concerne la sensibilité nociceptive au chaud pour laquelle la majorité des études concluent à une hypo-sensibilité [31-33, 35-41, 42]. Pour les sensibilités nociceptives thermique au froid, mécanique et viscérale, les résultats sont plus variables et sont fonction du modèle expérimental utilisé. L'utilisation de modèles de douleurs pathologiques indiquent cependant une réduction du développement des douleurs aiguës et chroniques, qu'elles soient d'origine inflammatoire ou neuropathique $[39,43,44]$.

Ces modèles ont par ailleurs permis de révéler certains des processus moléculaires impliqués (Figure 1). Des mécanismes généraux pouvant altérer le système nociceptif dans son ensemble, comme l'implication du système nicotinique ou une sur-activation des récepteurs NMDA du

${ }^{4}$ Ce modèle de souris BTBR se caractérise par des interactions sociales atypiques, des troubles de la communication et un comportement répétitif. glutamate, ont ainsi été décrits $[35,37]$. Des phénomènes plus localisés, au sein de structures impliquées dans l'intégration nociceptive, ont aussi été observés. Au niveau périphérique, des différences dans les seuils d'excitabilité des fibres $C$ ont été rapportées chez les animaux BTBR et $\mathrm{Fmrl}^{-/ y}$ [35]. La mutation du gène codant la protéine shank3, spécifiquement dans les neurones, pourrait également modifier le fonctionnement des canaux TRPVI dans les neurones sensoriels et ainsi diminuer la sensibilité nociceptive au chaud [43]. Concernant l'hypersensibilité mécanique, elle pourrait être liée à une augmentation de l'innervation au niveau de la peau [42] ou à une diminution d'expression des canaux potassiques de la famille HCN (hyperpolarisation-activated cyclic-nucleotid) qui augmenterait l'excitabilité des fibres mécano-sensibles [45].

Certaines études suggèrent également des altérations au sein du système nerveux central. Par exemple, après l'injection périphérique de formaline (un agent inflammatoire), la réponse nociceptive est typiquement constituée de deux phases : la première mettant en jeu l'activation des fibres périphériques, et la deuxième impliquant des phénomènes spinaux de sensibilisation centrale. Seule cette deuxième phase est réduite chez les animaux $\mathrm{Fmrl}^{-/-}$[44]. Chez les souris Shank2 ${ }^{-/-}$, la sensibilité des neurones de la corne dorsale de la moelle épinière à un agoniste des récepteurs NMDA du glutamate, semble être diminuée, réduisant les comportement de douleur [40]. De même, une diminution d'expression des récepteurs du GABA est observée dans la corne dorsale des souris Mecp2 ${ }^{-/-}$[28]. Ces modifications pourraient ainsi perturber la balance entre excitation et inhibition au niveau du réseau nociceptif spinal, entraînant des modifications majeures dans l'intégration des stimulus périphériques.

Au niveau cérébral, des atypies de transmission et de plasticité synaptiques ont été décrites. Elles impliquent, potentiellement, des changements morphologiques et fonctionnels et des déséquilibres de la balance excitation/inhibition au niveau du cortex somato-sensoriel ou de l'amygdale [36]. Ces modifications pourraient altérer les processus d'intégration et d'encodage des stimulus nociceptifs et ainsi perturber l'élaboration de la sensation de douleur.

L'activité de plusieurs molécules a pu être testée grâce à ces différents modèles animaux. Certaines se sont révélées avoir un potentiel thérapeutique intéressant dans les TSA. Des traitements ciblant les récepteurs nicotiniques de l'acétylcholine, les récepteurs GABA-A, ou les récepteurs NMDA du glutamate ont ainsi permis des améliorations des déficits sociaux et une réduction des comportements stéréotypés, ainsi qu'une amé- 


\begin{tabular}{|c|c|c|c|}
\hline $\begin{array}{l}\text { Espèce, Sexe, } \\
\text { Modèle }\end{array}$ & $\begin{array}{l}\text { Modalité thermique } \\
\text { au chaud (1) }\end{array}$ & $\begin{array}{l}\text { Modalité thermique } \\
\text { au froid ( } 2 \text { ) }\end{array}$ & Modalité mécanique (3) \\
\hline $\begin{array}{l}\text { Rat } 0^{2} \\
\text { VPA }\end{array}$ & Hypo-sensibilité & - & Hyper-sensibilité (période juvénile seulement) \\
\hline $\begin{array}{l}\text { Souris } \sigma^{\prime} \\
\text { VPA }\end{array}$ & Hypo-sensibilité & - & - \\
\hline $\begin{array}{l}\text { Souris } 0^{7} \\
\text { Syngap } 1^{-/+}\end{array}$ & Hypo-sensibilité & - & - \\
\hline $\begin{array}{l}\text { Souris O' } \\
\text { Shank2 }\end{array}$ & Hypo-sensibilité & - & Hypo-sensibilité \\
\hline 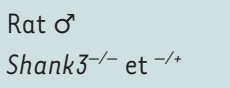 & Hypo-sensibilité & - & Hypo-sensibilité \\
\hline $\begin{array}{l}\text { Souris o" } \\
\text { Shank } 3^{-/-} \text {et }^{-/+}\end{array}$ & Normo-sensibilité & Normo-sensibilité & Normo-sensibilité \\
\hline $\begin{array}{l}\text { Rat } \sigma^{\top} \\
\text { Mecp2-/y }\end{array}$ & Hypo-sensibilité & Hyper-sensibilité & Hyper-sensibilité \\
\hline $\begin{array}{l}\text { Rat } 9 \\
\text { Mecp2-/x }\end{array}$ & Normo-sensibilité & Hyper-sensibilité & Hyper-sensibilité \\
\hline $\begin{array}{l}\text { Souris } 0^{7} \\
\text { Fmrl }{ }^{-/ y}\end{array}$ & Hypo-sensibilité & Hypo-sensibilité & - \\
\hline $\begin{array}{l}\text { Souris } \sigma^{x} \\
\text { Fmrl }^{-/ y}\end{array}$ & Normo -sensibilité & - & Normo -sensibilité \\
\hline $\begin{array}{l}\text { Souris ơㅇ } \\
\text { Gabrb3 } 3^{-/+}\end{array}$ & Hyper-sensibilité & - & $\begin{array}{l}\text { Hyper-sensibilité } \\
\text { (ơ seulement) }\end{array}$ \\
\hline $\begin{array}{l}\text { Souris ơㅇ } \\
\text { BTBR }\end{array}$ & Hypo-sensibilité & Hypo-sensibilité & - \\
\hline
\end{tabular}

Tableau II. Sensibilité nociceptive dans les modèles animaux d'autisme. La sensibilité nociceptive thermique est testée par l'observation de comportements nociceptifs (retrait de la patte ou de la queue, léchage, secousse) lors d'exposition à une température fixe $\left(52^{\circ} \mathrm{C}\right.$ ou $\left.0^{\circ} \mathrm{C}\right)$ ou $\mathrm{croissante}(30-$ $52^{\circ} \mathrm{C}$ ) pour le chaud et décroissante $\left(25-0^{\circ} \mathrm{C}\right)$ pour le froid. Le seuil de sensibilité mécanique est mesuré en appliquant au niveau de la patte arrière de l'animal une force croissante à l'aide de filaments en nylon de diamètre calibrés (filaments de von Frey) ou à l'aide de pinces calibrées en observant un éventuel retrait du membre. La sensibilité viscérale peut être évaluée en observant les comportements consécutifs à une injection intrapéritonéale d'acide acétique (grattages abdominaux, étirement des pattes postérieures, torsions de la musculature dorso-abdominale) ou par distension colorectale à l'aide d'un ballon et enregistrement électromyographique de la réponse viscéro-motrice. Le modèle de douleur aiguë évoquée par la capsaïcine consiste 


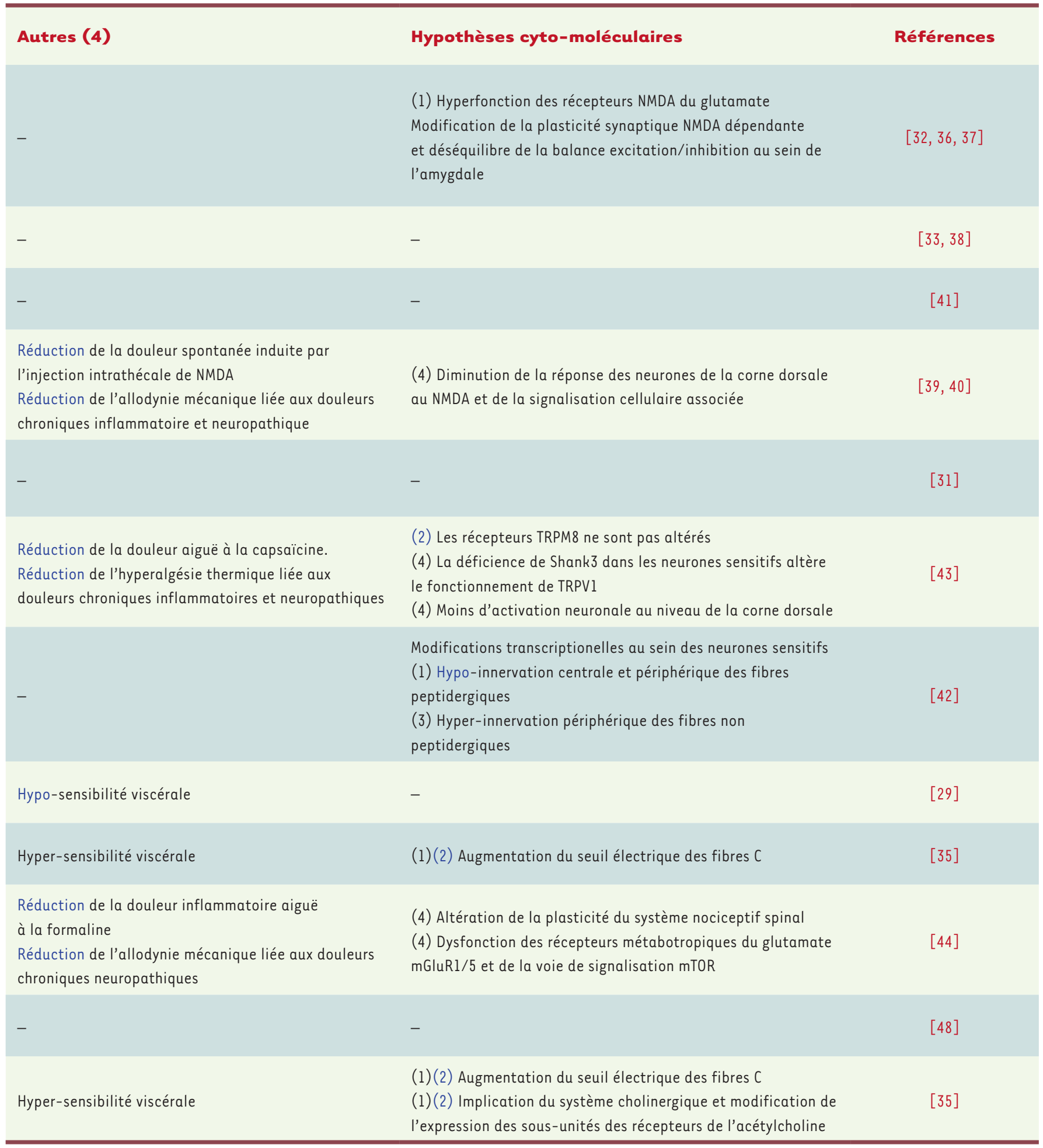

en l'injection intraplantaire d'un agoniste des récepteurs TRPVI (transient receptor potential vanilloid l), et à l'observation des comportements nociceptifs spontanés. Le modèle de douleur inflammatoire aiguë à la formaline repose sur un principe similaire avec l'injection d'un agent chimique pro-inflammatoire qu'est la formaline. La réponse présente deux phases, la première mettant en jeu une activation directe des fibres périphériques et la deuxième phase, plus tardive, mettant en jeu des phénomènes de sensibilisation centrale au niveau de la moelle épinière. Le modèle de douleur chronique de type inflammatoire repose sur l'injection intraplantaire d'un agent pro-inflammatoire comme l'adjuvant complet de Freund alors que le type neuropathique nécessite une chirurgie préalable pour procéder à une ligature, une compression ou une section de certaines racines nerveuses du nerf sciatique. 
lioration des atypies somato-sensorielles, notamment nociceptives $[35,37,45]$. Le bumétanide, un traitement très prometteur dans les TSA, en restaurant l'équilibre excitation/inhibition, semble également corriger les altérations de la sensibilité somato-sensorielle [46].

\section{Conclusions et perspectives}

Le traitement de l'information nociceptive et la sensation de douleur qui peut en découler présentent des particularités chez les sujets autistes. Sachant qu'ils sont plus souvent confrontés à des situations douloureuses que la population générale, ces particularités sont à prendre en considération afin d'améliorer leur qualité de vie. Cependant, les observations réalisées chez ces patients sont diverses, vraisemblablement en raison des différences de sévérité de leur autisme, de leurs capacités intellectuelles et de leur profil sensoriel qui est propre à chaque individu. Ainsi, des tests standardisés et plus adaptés aux particularités cognitives et sensorielles des patients porteurs de troubles du spectre autistique et à l'hétérogénéité de cette maladie pourraient permettre de mieux appréhender la nature de ces altérations. Bien qu'il soit actuellement difficile d'établir des corrélations entre l'homme et les modèles animaux, le recours à ces derniers paraît pertinent. Ils permettraient de préciser les modalités nociceptives altérées, de comprendre les mécanismes cellulaires et moléculaires impliqués et de tester des molécules susceptibles de corriger les altérations sensorielles des sujets autistes. $\diamond$

\section{SUMMARY}

\section{Nociception pain and autism}

Autistic subjects frequently display sensory anomalies. Those regarding nociception and its potential outcome, pain, are of crucial interest. Indeed, because of numerous comorbidities, autistic subjects are more often exposed to painful situation. Despite being often considered as less sensitive, experimental studies evaluating this point are failing to reach consensus. Using animal model can help reduce variability and bring, regarding autism, an overview of potential alterations of the nociceptive system at the cellular and molecular level. $\diamond$

\section{LIENS D'INTÉRÊT}

Les auteurs déclarent n'avoir aucun lien d'intérêt concernant les données publiées dans cet article.

\section{RÉFÉRENCES}

1. American Psychiatric Association. Diagnostic and statistical manual of mental disorders (DSM5), $5^{\text {th }}$ ed. Arlington, VA : APA, 2013.

2. Thye MD, Bednarz HM, Herringshaw AJ, et al. The impact of atypical sensory processing on social impairments in autism spectrum disorder. Dev Cogn Neurosci 2018 ; 29 : 151-67.

3. Moore DJ. Acute pain experience in individuals with autism spectrum disorders: a review. Autism $2015 ; 19$ : 387-99.

4. Summers J, Shahrami A, Cali S, et al. Self-injury in autism spectrum disorder and intellectual disability: exploring the role of reactivity to pain and sensory input. Brain Sci 2017 ; $7: 1-16$.

5. Bourne S, Machado AG, Nagel SJ. Basic anatomy and physiology of pain pathways. Neurosurg Clin North Am $2014 ; 25: 629-38$.

6. Woolf CJ, Ma Q. Nociceptors-noxious stimulus detectors. Neuron 2007 ; 55 : 353-64.

7. Cordero-Erausquin M, Inquimbert P, Schlichter R, et al. Neuronal networks and nociceptive processing in the dorsal horn of the spinal cord. Neuroscience $2016 ; 338: 230-47$.

8. Millan MJ. Descending control of pain. Prog Neurobiol $2002 ; 66: 355-474$.

9. Garcia-Larrea L, Peyron R. Pain matrices and neuropathic pain matrices: a review. Pain 2013 ; S29-43.
10. Wager TD, Atlas Ly, Lindquist MA, et al. An fMRI-based neurologic signature of physical pain. N Engl J Med 2013 ; 368 : 1388-97.

11. Fründt 0 , Grashorn W, Schöttle $D$, et al. Quantitative sensory testing in adults with autism spectrum disorders. J Autism Dev Disord 2017 ; 47 : 1183-92.

12. De Jonckheere J, Bonhomme V, Jeanne M, et al. Physiological signal processing for individualized anti-nociception management during general anesthesia: a review. Yearbook Medical Informatics 2015 ; 10 : 95-101.

13. Ely $\varepsilon$, Chen-Lim ML, Carpenter KM, et al. Pain assessment of children with autism spectrum disorders. J Dev Behav Pediatr 2016 ; 37 : 53-61.

14. Zabalia M, Breau LM, Wood C, et al. Validation francophone de la grille d'évaluation de la douleur-déficience intellectuelle: version postopératoire. Can J Anesth $2011 ; 58$ : 1016-23.

15. Dubois A, Michelon C, Rattaz C, et al. Daily living pain assessment in children with autism: Exploratory study. Res Dev Disabil 2017 ; 62 : 238-46.

16. Tordjman S, Anderson GM, Botbol M, et al. Pain reactivity and plasma $\beta$-endorphin in children and adolescents with autistic disorder. PLoS One $2009 ; 4:$ e 5289.

17. Cascio C, McGlone F, Folger S, et al. Tactile perception in adults with autism: a multidimensional psychophysical study. J Autism Dev Disord 2008 ; 38 : 127-37.

18. Yasuda $Y$, Hashimoto R, Nakae A, et al. Sensory cognitive abnormalities of pain in autism spectrum disorder: A case-control study. Ann Gen Psychiatry $2016 ; 15: 8$.

19. Vaughan S, McGlone F, Poole H, et al. A quantitative sensory testing approach to pain in autism spectrum disorders. J Autism Dev Disord 2019; $50: 1607-20$.

20. Duerden EG, Taylor MJ, Lee M, et al. Decreased sensitivity to thermal stimuli in adolescents with autism spectrum disorder: relation to symptomatology and cognitive ability. J Pain $2015 ; 16: 463-71$.

21. Chien YL, Wu SW, Chu CP, et al. Attenuated contact heat-evoked potentials associated with sensory and social-emotional symptoms in individuals with autism spectrum disorder. Sci Rep $2017 ; 7: 36887$.

22. Failla MD, Moana-Filho $\varepsilon$ J, Essick GK, et al. Initially intact neural responses to pain in autism are diminished during sustained pain. Autism $2018 ; 22$ : 669-83.

23. Riquelme I, Hatem SM, Montoya P. Abnormal pressure pain, touch sensitivity, proprioception, and manual dexterity in children with autism spectrum disorders. Neural Plast 2016; 2016 : 1723401.

24. Fan YT, Chen C, Chen SC, et al. Empathic arousal and social understanding in individuals with autism: evidence from $\mathrm{FMRI}$ and $\varepsilon R P$ measurements. Soc Cogn Affect Neurosci $2014 ; 9$ : 1203-13.

25. Orefice LL. Peripheral somatosensory neuron dysfunction: emerging roles in autism spectrum disorders. Neuroscience $2020 ; 445: 120-9$.

26. Donovan APA, Basson MA. The neuroanatomy of autism: a developmental perspective. J Anatomy $2017 ; 230: 4-15$.

27. Riquelme I, Hatem SM, Montoya P. Reduction of pain sensitivity after somatosensory therapy in children with autism spectrum disorders.J Abnorm Child Psychol $2018 ; 46$ : 1731-40.

28. Orefice LL, Zimmerman AL, Chirila AM, et al. Peripheral mechanosensory neuron dysfunction underlies tactile and behavioral deficits in mouse models of ASDs. Cell $2016 ; 166: 299-313$.

29. Bhattacherjee A, Winter MK, Eggimann LS, et al. Motor, somatosensory, viscerosensory and metabolic impairments in a heterozygous female rat model of rett syndrome. Int J Mol Sci $2018 ; 19$ : 97.

30. Das I, Estevez MA, Sarkar AA, et al. A multifaceted approach for analyzing complex phenotypic data in rodent models of autism. Mol Autism 2019; $10: 11$.

31. Song TJ, Lan XY, Wei MP, et al. Altered behaviors and impaired synaptic function in a novel rat model with a complete Shank3 deletion. Front Cell Neurosci $2019 ; 13: 111$.

32. Schneider T, Przewłocki R. Behavioral alterations in rats prenatally to valproic acid: animal model of autism. Neuropsychopharmacology 2005 ; $30: 80-9$.

33. Castro K, Baronio D, Perry IS, et al. The effect of ketogenic diet in an animal model of autism induced by prenatal exposure to valproic acid. Nutr Neurosci $2017 ; 20: 343-50$.

34. Bossu JL, Roux S. Les modèles animaux d'étude de l'autisme : le modèle valproate. Med Sci 2019 ; 35 : 236-43.

35. Wang L, Almeida LEF, Nettleton M, et al. Altered nocifensive behavior in animal models of autism spectrum disorder: The role of the nicotinic cholinergic system. Neuropharmacology 2016 ; 111 : 323-34. 


\section{RÉFÉRENCES}

36. Wu HF, Chen PS, Chen YJ, et al. Alleviation of $\mathrm{N}$-methyl-d-aspartate receptor-dependent longterm depression via regulation of the glycogen synthase kinase- 3 pathway in the amygdala of a valproic acid-induced animal model of autism. Mol Neurobiol 2017 ; 54 : 5264-76.

37. Mohammadi S, Asadi-Shekaari M, Basiri M, et al. Improvement of autistic-like behaviors in adult rats prenatally exposed to valproic acid through early suppression of NMDA receptor function. Psychopharmacology $2020 ; 237: 199-208$

38. Baronio D, Castro K, Gonchoroski T, et al. Effects of an H3R antagonist on the animal model of autism induced by prenatal exposure to valproic acid. PLoS One 2015; 10 : e0116363.

39. Ko HG, Oh SB, Zhuo M, et al. Reduced acute nociception and chronic pain in Shank $2^{-1-}$ mice. Mol Pain 2016; 12 : 1744806916647056 .

40. Yoon SY, Kwon SG, Kim YH, et al. A critical role of spinal Shank2 proteins in NMDA-induced pain hypersensitivity. Mol Pain 2017 ; 13 : 1-9.

41. Nakajima R, Takao K, Hattori S, et al. Comprehensive behavioral analysis of heterozygous Syngapl knockout mice. Neuropsychopharmacol Reports 2019 ; 39 : 223-37.

42. Bhattacherjee $\mathrm{A}, \mathrm{Mu} Y$, Winter MK, et al. Neuronal cytoskeletal gene dysregulation and mechanical hypersensitivity in a rat model of Rett syndrome. Proc Natl Acad Sci USA 2017 ; 114 : E6952-61.

43. Han $Q$, Kim $Y H$, Wang $X$, et al. SHANK3 deficiency impairs heat hyperalgesia and trpvl signaling in primary sensory neurons. Neuron $2016 ; 92: 1279-93$.
44. Price TJ, Rashid MH, Millecamps M, et al. Decreased nociceptive sensitization in mice lacking the fragile $\mathrm{X}$ mental retardation protein: role of $\mathrm{mGluRl} / 5$ and mTOR. J Neurosci $2007 ; 27: 13958-67$

45. Orefice LL, Mosko JR, Morency DT, et al. Targeting peripheral somatosensory neurons to improve tactile-related phenotypes in ASD models. Cell 2019 $178: 867-86$.

46. He Q, Arroyo ED, Smukowski SN, et al. Critical period inhibition of NKCCl rectifies synapse plasticity in the somatosensory cortex and restores adult tactile response maps in fragile X mice. Mol Psychiatry 2019 ; $24: 1732-47$.

47. Bird G, Silani G, Brindley R, et al. Empathic brain responses in insula are modulated by levels of alexithymia but not autism. Brain $2010 ; 133: 1515$ 25.

48. DeLorey TM, Sahbaie P, Hashemi $\varepsilon$, et al. Somatosensory and sensorimotor consequences associated with the heterozygous disruption of the autism candidate gene, Gabrb3. Behav Brain Res 2011 ; 216 : 36-45.

\section{TIRÉS À PART}

A. Furlan

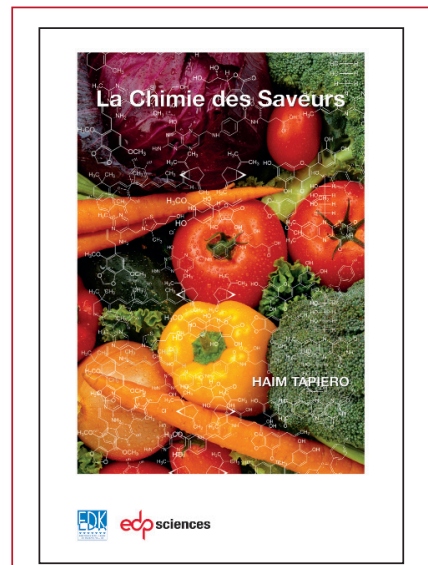

a cuisine est une science. II existe une relation étroite entre élaborer une recette et entreprendre une recherche scientifique. Quelle que soit l'origine d'une recette, d'un livre ou inventée, il faudra faire le choix des ingrédients, les mélanger et les cuire de manière appropriée afin de ne pas altérer les substances actives qui composent les ingrédients.

Une fois la cuisson terminée, il faudra analyser le goût et si nécessaire prévoir son amélioration. Améliorer une recette nécessite de connaître le ou les processus qui interviennent dans le développement des arômes, des saveurs et de la texture. Cette approche est similaire à celle développée par le scientifique.

La relation entre l'élaboration des recettes, les substances nutritives qui composent les ingrédients et la santé de l'homme est issue de plusieurs disciplines de la recherche fondamentale et clinique. Au cours des dernières années, de nombreux travaux scientifiques ont été publiés sur le rôle de la nutrition et la réduction des risques dans les pathologies comme les maladies cardio-vasculaires ou les cancers.

Le but principal de cet ouvrage a été d'identifier la structure chimique des composants actifs des ingrédients utilisés en cuisine (légumes, herbes aromatiques, épices) et qui entrent dans la préparation des recettes pour « végétariens » et « omnivores ».

ISBN : 978-2-7598-1137-3 180 pages

À retourner à EDP Sciences, 17 avenue du Hoggar, 91944 Les Ulis Cedex A - E-mail : francois.flori@edpsciences.org

NOM :

Prénom

Adresse :

Code postal :

Ville :

Pays :

Fonction

Je souhaite recevoir l'ouvrage La chimie des Saveurs : $20 €+3 €$ de port $=\mathbf{2 3} €$ TTC

en ................ exemplaire, soit un total de $€$

$\square$ Par chèque, à l'ordre de EDP Sciences

$\square$ Par carte bancaire :

$\square$ Visa

Signature :

Date d'expiration :

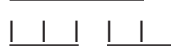

$\mathrm{N}^{\circ}$ de contrôle au dos de la carte :

$1|1|$

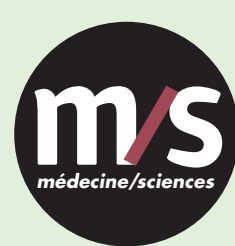

Tarifs d'abonnement $\mathrm{m} / \mathrm{s}$ - 2020

$>$ Grâce à $m / s$, vivez en direct les progrès des sciences biologiques et médicales

Abonnez-vous

à médecine/sciences

Bulletin d'abonnement page 202 dans ce numéro de $\mathrm{m} / \mathrm{s}$
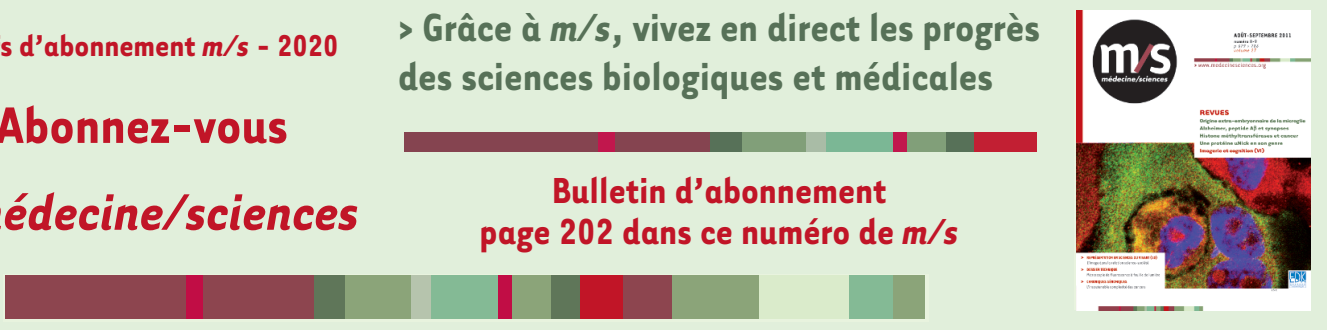